\title{
Shunting inhibition, a silent step in visual cortical computation
}

\author{
Yves Frégnac * , Cyril Monier, Frédéric Chavane, Pierre Baudot, Lyle Graham \\ Unité de Neurosciences Intégratives et Computationnelles, UPR CNRS 2191, Institut de Neurobiologie Alfred Fessard, Bat. 33, \\ 1 Avenue de la Terrasse, Gif-sur-Yvette 91198, France
}

\begin{abstract}
Brain computation, in the early visual system, is often considered as a hierarchical process in which features extracted in a given sensory relay are not present in previous stages of integration. In particular, orientation preference and its fine tuning selectivity are functional properties shared by most cortical cells and they are not observed at the preceding geniculate stage. A classical problem is identifying the mechanisms and circuitry underlying these computations. Several organizational principles have been proposed, giving different weights to the feedforward thalamocortical drive or to intracortical recurrent architectures. Within this context, an important issue is whether intracortical inhibition is fundamental for the genesis of stimulus selectivity, or rather normalizes spike response tuning with respect to other features such as stimulus strength or contrast, without influencing the selectivity bias and preference expressed in the excitatory input alone. We review here experimental observations concerning the presence or absence of inhibitory input evoked by non-preferred orientation/directions. Intracellular current clamp and voltage clamp recordings are analyzed in the light of new methods allowing us (1) to increase the visibility of inhibitory input, and (2) to continuously measure the visually evoked dynamics of input conductances. We conclude that there exists a diversity of synaptic input combinations generating the same profile of spike-based orientation selectivity, and that this diversity most likely reflects anatomical non-homogeneities in input sampling provided by the local context of the columnar and lateral intracortical network in which the considered cortical cell is embedded.

(C) 2004 Published by Elsevier Ltd.
\end{abstract}

Keywords: Visual cortex; Orientation selectivity; Excitation; Inhibition; Conductance; Voltage clamp; Feedforward; Recurrent; High conductance state

\section{Introduction}

A prevailing concept in the role of thalamocortical pathways in sensory processing is the dominant influence of feedforward connectivity. In the case of the mammalian visual system, it is well established that topographic maps and the organization of visual receptive fields in $\mathrm{ON}$ and $\mathrm{OFF}$ discharge zones result from the strong imprint of the feedforward input ([27] review in [24]). Hierarchical models of visual processing assume that the spatial convergence of afferents from one relay to the next determines the functional architecture of the target structure. For example, these models predict that the unique selectivity of the cortical cell firing to oriented contours found in primary visual

\footnotetext{
${ }^{*}$ Corresponding author. Tel.: +33-1-69-82-34-15; fax: +33-1-69-8234-27.

E-mail address: yves.fregnac@iaf.cnrs-gif.fr (Y. Frégnac).
}

cortex is obtained by the precise combination of inputs arising from geniculate cells of the same type (ON or OFF) whose receptive fields are offset and aligned along a given axis in the visual field. Electrophysiological correlates have been reported at least in the thalamic input recipient layer in ferret and cat visual primary cortex $[11,37]$.

Such a view, which requires extreme precision in the wiring of extrinsic thalamic afferents to primary sensory areas, may also apply to interareal connections at a higher level of cortical processing. Recent evidence, often interpreted as supporting the serial feedforward nature of processing up to perceptual decision centers, comes from latency measurements of scalp event-related potentials in frontal cortical areas. Changes in the electrical activity correlated with complex cognitive tasks, such as deciding on the animal vs. non-animal identity of briefly flashed images, can be detected as early as $150 \mathrm{~ms}$ following the image onset in the human and monkey observers [45]. This type of evidence seems 
to indicate, at least for certain perceptual tasks, a strong timing constraint that limits the involvement of lateral and recurrent cortical processing, given the number of synapses to be serially crossed along the cortico-cortical pathway. According to this schema, no time is left for the short- and long-distance intrinsic recurrent connections nor for the feedback from higher areas, except but to amplify or tune the functional bias set by the feedforward connectivity.

A fundamental issue in terms of computation is to understand whether this apparent feedforward dominance faithfully reflects the amount of intracortical processing, or if more complex spatio-temporal nonlinear interactions between feedforward and intracortical synaptic events have to be considered. We will review both models and electrophysiological data, which argue for an important role of local intracortical inhibitory processing, the effect of which is often hidden at the level of single cell spiking behaviour.

\section{Models of connectivity architecture in the primary visual cortex}

Although the various proposed models, whether based on experimental or theoretical ground, can be thought of as forming more a continuum than a set of distinct computation schemas, three basic types of architectures are generally considered and defined by the global activity regime of the cortex during sensory processing. The various subcircuits participating to these architectures are illustrated in Fig. 1.

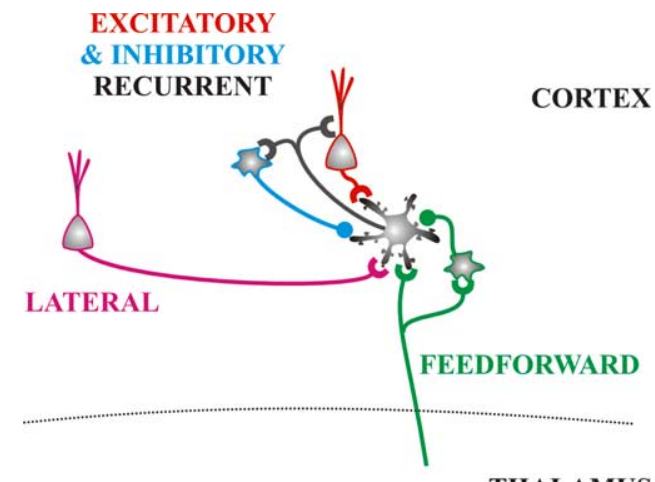

THALAMUS

Fig. 1. Schematic connectivity in primary visual cortex: an elementary circuit is illustrated, composed by a layer 4 spiny stellate cell receiving a direct thalamocortical input, two cortical pyramidal cells, and two inhibitory interneurons represented respectively by hairy circle, red triangles and smooth circles. Excitatory synapses are schematized by curved contours, whereas inhibitory synapses are represented by filled circles. Three types of connectivity are distinguished (see text: "feedforward" in green, including the monosynaptic excitatory thalamocortical input and a disynaptic inhibitory drive; "feedback", whether through recurrent excitatory (red) or inhibitory (blue) circuits; "lateral", originating from distinct cortical columns).

\section{1. "Feedforward" models}

The most basic connectivity is that provided by feedforward pathways from one defined anatomical level to the next, e.g. retina to thalamus, thalamus to layer IV cortical cells, interlaminar and interareal connections. Dating from the pioneering work of Hubel and Wiesel, many physiological and theoretical studies have suggested that cortical functional properties are either established earlier in the processing chain (e.g. thalamus for setting an orientation bias) or generated by highly specific patterns of synapses from subcortical levels to the next. For feedforward models of orientation selectivity, the computational role attributed to local intracortical connectivity is to simply propagate the orientation preference of layer IV neurons across a stereotyped intracortical microcircuit. These intrinsic connections, linking cells "vertically" within the same functional column, could eventually be recruited to elaborate other receptive field properties, such as length tuning, or build higher-order receptive field properties (such as position invariance of orientation preference within complex receptive fields). However, they would not modify the orientation preference imprinted by the pattern of geniculo-cortical inputs nor improve the selectivity of tuning. Long distance connections would also serve to establish horizontal links between territories with similar orientation preference, but their contribution to the tuning of the recipient cell is not considered in this connectivity framework as significant. This view has been recently supported by intracellular studies in cat area 17 demonstrating the functional dominance of the geniculate drive to layer IV cells. Orientation preference, and, to a certain degree, orientation tuning width remained invariant when the contribution from intracortical connections was artificially reduced. The silencing of postsynaptic activity in the primary visual cortex was obtained by either cooling the supragranular and middle layers of the cortex [23] or by inactivating the second-order cells with an electrical shock applied at the surface of the cortex [13]. Feedforward models have de-emphasized the functional role of intracortical circuitry, other than for perhaps one exception: since feedforward pathways are generally limited to excitatory synapses, local inhibitory neurons whose input is dominated by feedforward input may be considered as a part of this architecture. This parallel feedforward pathway basically implements a sign change on the input from the lower level. Consequently the "feedforward" concept was modified to include disynaptic intracortical inhibition as well as monosynaptic excitation in the "feedforward" drive to the cortex [24].

If feedforward models account for the dominant aspects in the spatial organization of simple receptive fields and their segregation in pure ON- and OFF-sub- 
fields, they fail to reproduce, in their simplest formulation, two of the most remarkable features of visual cortical receptive fields:

(i) The first feature is the high degree of selectivity in the orientation tuning of the spiking-based response, observed in most cortical cells when luminance gratings or light/dark bars are swept at constant speed across their receptive field. Purely linear excitatory convergence models cannot account for the restriction of the tuning width of cortical cells responses to a $15-30^{\circ}$ span on each side around the preferred orientation. Residual excitatory responses are indeed predicted for cross-oriented stimuli, due to the nonlinear rectification of the input signal by LGN cells, which is present for high contrast stimuli [40].

(ii) The second feature is the invariance of orientation tuning width, observed irrespectively of large variations in the contrast of the stimulus $[39,43]$. This property, specific of simple cells, also requires additional constraints on intracortical wiring. In order to account satisfactorily both for the spatial opponency and contrast gain control in simple receptive fields, the most recent generation of generalized "feedforward" models consists of a push-pull elementary circuit whose basic architecture consists of two pairs of cross-connected anti-phase excitatory and inhibitory simple receptive fields [46] to which a component of feedforward inhibition has been added. This inhibition is supposed to be provided by a specific set of inhibitory interneurons, with distinct properties, in the sense that their receptive field is complex, their responses are contrast-dependent and untuned to orientation [30]. Although tentative efforts are currently made to find physiological correlates of such units in layer IV [26], it remains contradictory, at least at the conceptual level, that the added cost in the implementation of contrast tuning width invariance is to assume the existence of an additional contingent of cells which precisely do not obey the searched property.

\subsection{Amplifier recurrent models}

The second type of architecture, often distinguished, is that of a "cortical amplifier", in which the contribution of the extensive intracortical lateral or local reafferent connections, which constitutes most of the synaptic input to layer IV stellate cells, is considered in full. A major distinction from the previous class of model is that the feedforward pathway does not impart strong selectivity on its own. Various models of this sort have emphasized the role of intracortical excitation, others the role of inhibition, now taken to be from interneurons that are fully embedded in the cortical network.
The emphasis here for the cortex would be to modulate the input/output properties of a given cell according to different computational tasks. In target identification, the cortical circuits would not so much decide on the orientation preference of a cortical cell, but rather on the gain necessary to recognize a given orientation independently of the stimulus contrast. In some other circumstances it may be important to up or down-regulate the level of response according to the peripheral context. Thus the proposed computational solution involves not simply a change in gain, but an adaptive resetting of the operating range according to the task. This view differs from that proposed by revised feedforward models in the sense that the amplifying operation is no longer local but recruits a larger set of neurons and synapses, creating some kind of network attractor ([4]; see also [1]). The group of Douglas and Martin described a canonical circuit where the variable gain of the cortical amplifier could be controlled by dominant feedback excitation characterized as a "network conductance" ([18,20]; see also [44]). Their model which makes use of both feedforward and feedback inhibition, qualitatively explains how "division" of excitation may occur in an assembly composed of linear-like neurons: feedback inhibition, acting through recurrent "subtractive" synapses, also generates a network conductance that results in a divisive change in the spike discharge rate of the neuron [19].

\section{3. "Over-damped" models}

Several models attribute to inhibition a more decisive role than gain field control and amplification in the shaping of receptive field properties. They often assume different orientation selectivity and preference between excitatory and inhibitory input circuits (review in [47], based on electrophysiological evidence of some level of cross-oriented inhibition [34,41]) or a total absence of orientation bias in the inhibitory drive. In order to account for the functional suppression of feedforward excitation by concomitant inhibition, these models require either a strong enough level of feedforward inhibition or the existence of effective non-linear interactions between excitation and inhibition. The specific class of models which explicitly consider the inherent non-linearity of synaptic conductance modulation (e.g. "shunting" or "divisive" inhibition) may be called "over-damped", in the sense that the synaptic input to any given cell is generally strong enough so that the linear integrative properties of the neuron are relatively unimportant for predicting the dynamics of the cell's spike output. The over-damping consequences of the intracortical connectivity yield a divisive effect on the global feedforward gain (which would be observed if all intracortical loop contributions were cancelled). These models are characterized generally by a high level input 
conductance state. The non-linearity is best expressed when inhibition is both shunting and "silent", i.e. its presence is unnoticed in the voltage records when applied alone, but still acts in a divisive way when a concomitant excitatory input activates the cell [28]. A simple version is the so-called normalization model, which proposes a rescaling mechanism consisting of a feedback-driven inhibitory shunt whose strength is proportional to the average activity of a pool of excitatory neurons $[9,10]$. More elaborate theoretical approaches in visual cortex [32], as well as in somatosensory cortex [36], predict that high conductance states are present for both preferred and non-preferred stimuli, which raises the possibility that for non-preferred stimuli the membrane potential of cortical cells may be clamped by dominant inhibition.

\section{Implications for the genesis of orientation selectivity}

One important conclusion of the previous overview is that intracortical inhibition, whatever its mode of action-feedforward, lateral or feedback, whatever its effects-subtractive or divisive, may have been largely ignored or experimentally undetected. The diversity of the proposed models thus raises important questions concerning the respective roles that should be attributed to excitation and inhibition and the dynamics of their balance during visual processing. For some models functional selectivity is achieved by the temporal relationship between excitation and inhibition, being out of phase for preferred stimuli and in phase for non-preferred ones. In other models, inhibition, linear or not, overlaps with excitation for both preferred and nonpreferred stimuli, and the issue becomes a purely gain control problem. For each computational task, the weighting between feedforward and feedback connectivity has also to be determined. It remains highly plausible that, depending on the visual drive, the membrane potential of a cortical neurone may explore the full range of dynamic states from the dominance of the feedforward drive and low conductance states to that of strong feedback inhibition and high conductance states.

In order to further refine a model of visual cortex, more electrophysiological evidence at the level of elementary synaptic circuits is needed to answer two basic questions:

- How effective is inhibition in cancelling the effects of visually evoked excitation?

- Is there a fixed canonical combination of excitatory and inhibitory inputs for the genesis of orientation and direction selectivity?

We will examine evidence from our laboratory [33], based on a large number of intracellular recordings, including both patch and sharp electrodes, in cat area 17 in vivo, which suggests that models of cortical processing should consider both a fundamental role of intracortical inhibition (and thus of high input conductance states), and a high diversity of combinations of synaptic inputs rather than a single fixed canonical circuitry for the computation of orientation and direction selectivity.

\section{The revival of shunting inhibition in vivo}

To date, experimental support for the contribution of inhibition in visual cortical receptive field properties has been somewhat contradictory. The strongest support has been obtained by electrophysiological protocols augmented by pharmacological manipulations. It has long ago been demonstrated in cat primary visual cortex that $\mathrm{GABA}_{\mathrm{A}}$ antagonists can eliminate orientation and directional selectivity [42]. Related iontophoretic experiments, where GABA was applied exogenously to silence the input from columns distant from the recording site, similarly support suppressive interactions between neighbouring columns differing in orientation preference [14]. However, attempts to block $\mathrm{GABA}_{\mathrm{A}}$ receptors intracellularly appeared not to change the qualitative tuning properties of the cell [35], although some unavoidable technical caveats may require further re-examination.

More recently, and probably because of the refinement of intracellular patch recording techniques in vivo, a growing number of experimenters have confirmed the long awaited [28] but long denied (e.g. [5,17,22]) existence of "shunting" inhibition in vivo. Our own in vivo data were the first to provide a quantitative demonstration at the primary visual cortex level that $\mathrm{GABA}_{\mathrm{A}}$ input may interact non-linearly with excitatory input during sensory processing [6,7]. In order to demonstrate the shunt action unambiguously, we studied the dynamics of input conductance triggered by the onset of a visual stimulus for stimuli flashed at the optimally orientation, whose position (spatial phase) was varied across the receptive field width. The rationale of the experiments was the following: depending on the electrotonic architecture of the neuron, and the locations of its inputs relative to the site of action potential generation, shunting by $\mathrm{GABA}_{\mathrm{A}}$-mediated inhibition of visually evoked excitatory postsynaptic potentials (EPSPs) should increase the neuron input conductance, $G(t)$, on the order of $50-100 \%$ or greater [29] (when compared to the resting condition, $G_{0}$ ) to have a sizeable suppressive effect. This range of conductance changes is required for $\mathrm{GABA}_{\mathrm{A}}$-mediated inhibition to have a functional contribution to properties such as the spatial separation of $\mathrm{ON}$ and OFF responses, and orientation or direction tuning. To measure this modulation, we used whole cell patch recordings in voltage clamp mode, that allowed 
the estimation of both $G(t)$ and the apparent reversal potential driving the composite synaptic input, $E_{\mathrm{rev}}(t)$, as continuous functions of time (Fig. 2). The results showed that an early shunt signal was often visible (mostly in simple cells) after the start of the initial postsynaptic depolarization of cortical cells, which corresponds to a transient increase in somatic conductance. This increase was seen both for preferred (optimal spatial phase) and non-preferred (opponent) flashed stimuli indicating that excitation is most often accompanied in Simple receptive fields by different levels of inhibition depending on the stimulus features. The intracortical synaptic events associated with the conductance increase appear to be dominated by $\mathrm{GABA}_{\mathrm{A}}$ receptor activation, since the $E_{\text {rev }}$ value associated with the conductance increase peak value was around $-65 \mathrm{mV}$. Our data suggest that visual cortical cells often operate in a high conductance mode, and thus their membrane potential tracks the apparent composite reversal potential corresponding to the balance between excitation and inhibition [32]. The key determinant of the spiking behaviour of the postsynaptic cell is the position of this composite reversal potential relative to the spike initiation threshold, expressing the functional clamp exerted by the network.

The continuous measurement of visually evoked conductance dynamics avoids several limitations of previous methods, such as measuring the amplitude

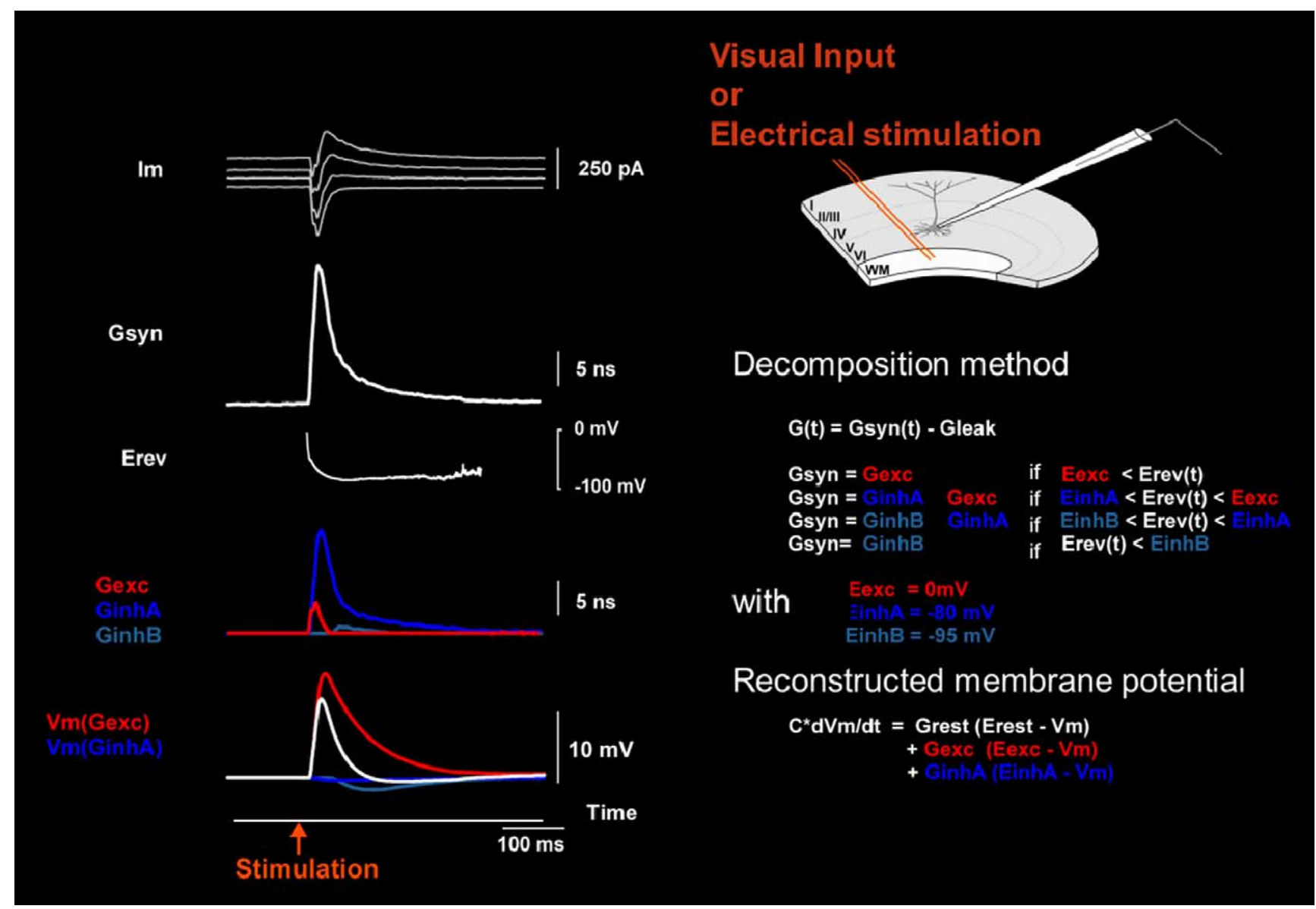

Fig. 2. Continuous measurement of input conductance. Top left panel: intracellular voltage clamp recordings. Stimulation-locked profiles of current traces are represented for different holding potentials ranging from -60 to $-90 \mathrm{mV}$. A current-voltage analysis (detailed in Ref. [36]), performed at various delays following the stimulation onset, allows the reconstruction of the stimulus evoked dynamics of the global input conductance $(G(t))$ and of the associated composite reversal potential $\left(E_{\mathrm{rev}}\right)$. Note that the peak of conductance is observed when $E_{\mathrm{rev}}$ becomes dominated by the inhibitory drive. Right panel: The conductance waveform is decomposed to give the sum of a constant leak conductance $\left(G_{\text {leak }}\right)$ and a global synaptic conductance $\left(G_{\mathrm{syn}}\right)$. The synaptic conductance term is further linearly decomposed into three conductance components corresponding to the activation of one type of excitatory synapse $(0 \mathrm{mV})$ and two types of inhibitory synapses, each associated with fixed reversal potentials $(-80 \mathrm{mV}$ for $\mathrm{GABA}$ and $-95 \mathrm{mV}$ for $\mathrm{GABA}_{\mathrm{B}}$ receptors). We made the additional hypothesis that, depending on the actual value of the composite synaptic reversal potential, one or two out of three possible types of inputs contribute in a dominant manner to synaptic activation. Lower left panel: The synaptic conductance components obtained by the decomposition method are overlaid on the same graph, with $G_{\text {exc }}$ in red, $G_{\text {inh }}\left(\mathrm{GABA}_{\mathrm{A}}\right)$ in blue and $G_{\text {inh }}\left(\mathrm{GABA}_{\mathrm{B}}\right)$ in green. In the bottom left graph, the changes in membrane potential produced by the individual activation of each synaptic component are compared with the global reconstituted membrane potential change. The shunting effect can be estimated by comparing the reconstituted profile (white curve) with the linear combination of the three synaptic components. 
modulation of responses applied in current clamp either to injected current pulses (e.g. [35]) or of electrically evoked thalamocortical EPSPs during the sensory (visually) evoked response [17]. Our findings of large conductance increases evoked by optimally oriented flashed stimuli were reproduced by other laboratories, using the same continuous measurement method under current clamp conditions [2,25]. However, one should keep in mind that, for cells in which we could perform both types of measurement, the absolute increase in conductance was larger when derived from voltage clamp than current clamp data, reflecting the fact that the first method is less affected by intrinsic membrane voltage-dependent non-linearities than the latter one.

\section{For a diversity of combinations of excitatory and inhibitory inputs}

In order to explain the genesis of orientation selectivity, the dominant view up to now has been to assume that the feedforward input generates similar levels of orientation selectivity in the excitatory and inhibitory drives (same orientation preference and tuning width), and therefore that intracortical inhibition participates in neither the setting nor the refining of orientation selectivity [24]. However the evidence underlying this consensus is scarce in terms of cell sample size and concerns a subpopulation of cortical cells, namely layer IV simple cells. Since in theory, there is a variety of possible combinations of the tuning of excitatory and inhibitory synaptic inputs, relative to the output firing tuning, larger samples of cortical cells may be required to ascertain the existence of specific combinations of excitation and inhibition.

We thus decided to quantitatively re-examine this issue, using both sharp and patch electrodes, in order to complement the advantages of each technique applied in the same in vivo preparation. Cells were recorded intracellularly in the primary visual cortex of adult anaesthetized (Althesin) and paralyzed cats. Sharp electrode recordings were performed in bridge mode with 55-90 M $\Omega$ glass pipettes filled with $2 \mathrm{M}$ potassium methyl sulfate and $4 \mathrm{mM}$ potassium chloride. whole cell patch recordings were made with 3-5 $\mathrm{M} \Omega$ glass patch electrodes filled with a solution containing $140 \mathrm{mM} \mathrm{K-}$ gluconate, $10 \mathrm{mM}$ HEPES, $4 \mathrm{mM}$ ATP, $4 \mathrm{mM} \mathrm{MgCl}_{2}$, $0.4 \mathrm{mM}$ GTP and $0.5 \mathrm{mM}$ EGTA $(\mathrm{KOH})(\mathrm{pH}$ adjusted to 7.3 with $\mathrm{KOH}$ and the osmolarity adjusted to 285 mosM). The seal resistance in attached mode was always above $1 \mathrm{G} \Omega$. In voltage clamp recordings, the access resistance was always lower than $40 \mathrm{M} \Omega$. Recordings were not restricted to layer IV and included both Simple and Complex cells. The rationale for this global sampling is that whatever the hierarchical status of the cell in the processing flow, the strongest part of the spike response is evoked at latencies at which intracortical and feedback activity are inevitably put into play.

Three experimental situations were considered:

- We used current clamp measurements made with sharp and patch electrodes to determine how often hyperpolarizing responses to cross-oriented stimuli could be detected in the resting condition. The resting potential of cortical cells is usually more depolarized by $5-15 \mathrm{mV}$ than the reversal potential for $\mathrm{GABA}_{\mathrm{A}}$ receptor activation and by $20-30 \mathrm{mV}$ than that for $\mathrm{GABA}_{\mathrm{B}}$ receptor activation. Consequently one should expect that the dominant presence of visually driven inhibition, when it is not masked by concomitant excitation, should be signalled by an evoked hyperpolarization.

- In a second study, we devised a new protocol allowing us to improve the detectability of inhibitory input even in the absence of excitation. Visual responses were measured when the cell was kept artificially depolarized ( -40 to $-20 \mathrm{mV}$ ) by a constant intracellular current injection. The intensity of this current was titrated for each cell, such as to inactivate fast sodium channels. This had the advantage of suppressing spike activity while increasing the driving force of inhibitory events, making them detectable as hyperpolarizations of larger amplitude than those normally evoked from the resting state.

- Finally, whole cell patch clamp recordings were used to monitor evoked synaptic currents in continuous voltage clamp mode and trace the continuous dynamics of the input conductance as a function of the stimulus orientation and position across the visual field. The measures of $G(t)$ and $E_{\text {rev }}(t)$ that we have realized using voltage clamp recordings allow first-order estimates of excitatory and inhibitory synaptic inputs. This is done by assuming a simple lumped circuit neuron model comprised of a parallel combination of a leak conductance, an excitatory synapse and two inhibitory $\mathrm{GABA}_{\mathrm{A}}$ and $\mathrm{GABA}_{\mathrm{B}}$ synapses, each associated with known and fixed reversal potentials (see legend in Fig. 2). The tuning of the two types of input can be quantified by taking the integral of the derived synaptic waveforms over the entire response duration.

Our data, obtained from the order of one hundred cells, show that orientation preference of cortical cells expressed at the spiking level can be produced through various orientation/direction dependent combinations of excitatory and inhibitory inputs [33]. An important new finding was obtained by comparing the timecourse of the membrane voltage trajectories in current clamp over repeated trials. Subthreshold membrane potential changes evoked for non-preferred stimuli of- 


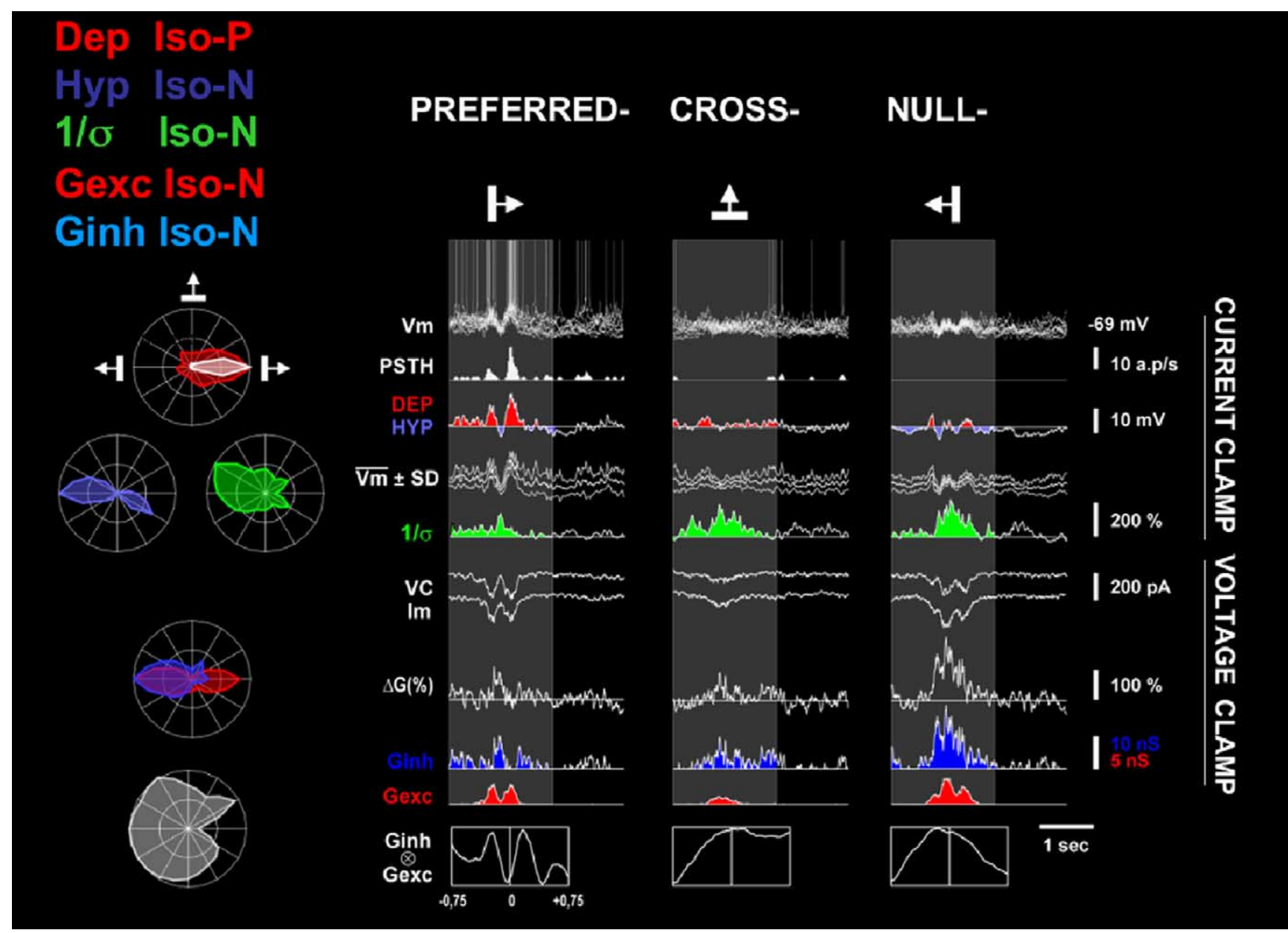

Fig. 3. Orientation and direction selectivity of excitatory and inhibitory inputs in visual cortical neurons. This figure presents one example of a whole cell patch recording, where measures of excitatory and inhibitory conductances were made both in current clamp (CC: upper panel) and voltage clamp (VC: lower part) modes. Current clamp recordings: From top to bottom, raw $V_{\mathrm{m}}$ recordings (depolarizing responses in red and hyperpolarizing responses in blue), mean and standard deviation of the stimulus-locked subthreshold waveform $(m \pm \sigma)$, and trial-to-trial variability reduction (green). The trial-to-trial variability reduction was calculated as the inverse of the standard deviation $(1 / \sigma)$ of the time-course of the stimulus-locked membrane potential response over all stimulation trials. The $1 / \sigma$ component was defined by the integral above the mean background value and the corresponding waveform (in green) is expressed as the percentage of change from the spontaneous reference level. Voltage clamp recordings: excitatory conductances are in red and inhibitory conductances in blue. Note that the scale for the excitatory conductance is double that for the inhibitory conductance. The bottom insets give the normalized cross-correlation function between the excitatory and inhibitory conductance waveforms and the ordinate for a zero time delay is used to quantify the degree of temporal overlap between the two waveforms. The illustrated cell has been recorded in whole cell patch. The receptive field (not shown here) is Simple. The preferred orientations of the various response components are respectively $2^{\circ}(\mathrm{Dep}), 169^{\circ}(\mathrm{Hyp}), 172^{\circ}(1 / \sigma), 180^{\circ}(\mathrm{Gexc})$ and $179^{\circ}(\mathrm{Ginh})$. Both synaptic conductances were strongest in the null direction.

ten showed a markedly reduced variance in the stimulus-locked membrane voltage waveform. This observation differed from the claim made in a previous report, where membrane potential variability was lumped across trials, and which did not find a significant dependency of the "noise" level in the membrane potential on the orientation and/or direction of the visual stimulus [3]. Our conclusion, based on current clamp recordings, is furthermore supported for cells in which voltage clamp conductance measurements could also be made: the time-course and the stimulus dependency in the reduction of the evoked $V_{\mathrm{m}}$ variability measured in current clamp mirrored the dy- namic signature of the change in inhibitory conductance measured in voltage clamp or in the spikeinactivated state.

Fig. 3 illustrates, for a typical cell, the methodology applied in our experiments and the systematic progression of the analysis from spike output (upper row) to input conductances (lower row). Current clamp recordings (CC), shown at the top of the figure, indicate that depolarization (red) evoked from rest is iso-tuned with the spike preference, whereas hyperpolarization (blue) is absent or weak for respectively cross-oriented (middle column) and null-direction stimuli (right column). However the detailed study of the variability 
of the stimulus-locked evoked membrane potential response shows strong variance reduction (in green) for both non-optimal (cross- and null-) stimuli, suggestive of a shunting effect of excitation by concomitant inhibition. The voltage clamp data obtained in the same cell (VC, middle traces) allow, using a limited set of assumptions, the reconstruction of the continuous profiles of the global input conductance (white curve) and of its excitatory (red filled curve) and inhibitory (blue filled curve) components evoked for each stimulus condition (see Methods in [33] and Fig. 2). We conclude in this particular cell that the direction/orientation selectivity profile of the response is generated by two dominant processes: (1) for the iso-preferred condition (left column), excitation and inhibition operate in a pushpull fashion, as quantified by the temporal correlation index between the two conductance waveforms which is indicative of an anti-phase behaviour (a negative peak value is observed for a zero shift value in the bottom left inset). (2) For non-optimal orientations/directions, excitation and inhibition act in phase and the silent shunting effect is demonstrated by the massive increase of inhibitory conductance for the null-stimuli. The most counter-intuitive finding in the chosen example is that the functional preference expressed by the firing rate of the cell is not due to the drive of a unidirectional preferred excitatory input, as one may have deduced from the current clamp recordings, but from the interplay of excitation and inhibition both pointing preferentially in the null direction. The temporal overlap between the excitatory and inhibitory conductance profiles and the associated shunting effect explain why no spike and evoked hyperpolarization are produced when the stimulus moves in the null direction. The out-of-phase behaviour between the two conductance profiles and the lesser direction selectivity of the excitatory input (compare red and blue tuning curves at the bottom left part of the figure) are responsible for the optimal firing observed when the stimulus moves in the $0^{\circ}$ "preferred" direction. This cell thus represents one of the cases where orientation/direction preference is produced by a combination of null-preferred excitation and null-preferred inhibition.

The same analysis extended to a larger set of cells demonstrates an unexpected level of diversity of combinations between excitation and inhibition. It suggests at the least that canonical models assuming iso-orientation preference for excitation and inhibition are largely oversimplified, and that "silent" shunting inhibition expressed for non-preferred directions and/or orientations should play a critical computational role in the genesis of functional preference and tuning in visual cortical cells. Two major combinations of synaptic inputs have been observed. One schema shows that the spike-based preference is dictated by that of the excitatory drive, but that the tuning width and polar asymmetry of the spike tuning is shaped by the inhibitory input whose orientation preference can be iso-, cross- or null-oriented. A second schema shows that the spike-based preference is unrelated to that of the excitatory and inhibitory inputs. However in this latter situation, both synaptic components systematically share the same off-peak orientation preference. In this latter case, the tuning of the spike response reflects non-homogeneities in the balance between excitation and inhibition, and is observed for the orientation range where excitation locally dominates over inhibition.

Several sources of non-homogeneity could contribute to the genesis of this functional diversity and are linked to the local intracortical connectivity context in which the cell is embedded. The first one is best viewed when projecting the input cell locations and the preference of their respective orientation column on the laminar plane. A recent study, combining intracellular recordings in the superficial layers of primary visual cortex and the concomitant optical imaging of the orientation network, concluded that there was a dependency of the input distribution on the location of the cell in the orientation and direction selective map [38]. In particular, cells located near to map singularities, such as pinwheels, would be in the vicinity of, and thus susceptible to be under the synaptic influence of, a larger spectrum of orientation preferences than cells immersed in the middle of isoorientation domains where all neighbours share the same orientation preference. Another source of diversity in the input distribution may be linked with the laminar position of the cell within the depth of the cortical column. Another study combining intracellular recordings and biocytin labelling reports that the tuning preference of the hyperpolarizing component (considered here as dominant or "net" inhibition) is correlated with the laminar location of cells [31]. This component was found to be dominantly iso-oriented for granular and supragranular layers, and often exhibiting a cross-oriented preference in deep layers, most specifically in layer V. In spite of the fact that they did not directly measure inhibitory conductances, these authors conclude that the inferred diversity in the inhibitory tuning seen across the cortical depth reflects hierarchical levels of integration in the serial flow of cortical processing. We propose here that the noticeable difference found between supragranular and granular layers on the one hand and infragranular layers on the other hand could simply reflect diversity in the input sampling in the laminar plane due to different axonal and dendritic lateral extension patterns. In summary, whatever organization axis of the network being considered, laminar or columnar, the diversity of input combinations found across cells reflects most likely the anatomical non-homogeneities in 
the connectivity context provided by the intracortical network.

\section{Spike timing and orientation selectivity controlled by a network consensus}

In contrast to the prediction of a localized synaptic integration field by purely feedforward schema, our earlier intracellular studies in cat area 17 show that the subthreshold receptive field may recruit spatially a much wider zone of the visual field than expected from the precision of feedforward projections $[8,12]$. An analysis of latencies separating the earliest signs of dominant visually evoked EPSPs and IPSPs and the spike output indicates that a time-window, much longer than previously thought, is available in many cells in the primary cortical network to process incoming input through excitatory and inhibitory recurrent and lateral connections. Subthreshold depolarizations in area 17 cortical neurons in response to flashed bars can be evoked with onset latencies as early as $18-20 \mathrm{~ms}$ whereas the first spike occurrence is generally observed at much longer delays $(>35 \mathrm{~ms})$. Our intracellular studies also show the existence of an early shunt signal dominated by $\mathrm{GABA}_{\mathrm{A}}$ receptor activation often visible after the start of the initial postsynaptic depolarization [7]. Thus a delay of up to several tens of milliseconds exists between the earliest signs of cortical activation and the mean latency of the output V1 signal.

Taking into account the recent description in area 17 of the cat of slow propagation of visual activity along excitatory and inhibitory lateral connections $[8,12]$, one may speculate that at least for certain stimulus features and even for specific classes of cells, local inhibitory circuits put other cortical cells on "hold", waiting for the confirmation or invalidation of the relevance of the input by the rest of the network. In order for such a schema to operate and to reconcile the apparent discrepancy between the onset latencies of depolarizing subthreshold events and of the first "visual" spike, we predict that a contingent of inhibitory cells is fired with a minimal delay of visual activation and clamps the network response through the action of shunting inhibition. The computational consequences of this "spike rephasing" process are multiple: (1) it could help to refine the temporal precision in spike activity patterns evoked in simple cells [16]. (2) It could serve contextual integration by imposing a delay for the authorization of the integration of converging excitatory input whatever its origin (feedforward, lateral, feedback) once a consensus between all the earlyactivated partners in the network has been reached on the relevance of the input for the receiving cell or column. This still hypothetical scenario shows that the timing constraints defined by feedforward models (see the introductory section) may have been simply overstated.

\section{Conclusion}

If such a view of input source multiplexing and delayed postsynaptic spiking holds in the temporal domain, a similar reasoning may apply in the orientation domain. The diverse but organized patterns of both short- and long-distance connectivity provided by the global orientation map makes it likely that cortical cells do not receive only iso-orientation input $[15,38]$. Consequently, in order to respect the functional identity of the cortical column, some integrative mechanism has to be thought of, whereby the cortical circuit tunes its gain for orientations close to that which will be the consensus preferred by the column. Our results indicate that there is no single recipe for the synaptic input distribution required to generate orientation selectivity: different cells show different combinations of the relative tuning of excitation and inhibition with respect to the spiking response. We propose the existence of a functional "map conformation" process which tends to realign the orientation preference of a cortical cell with that dictated by its immediate neighbourhood in the orientation map, irrespectively of the individual bias provided by the feedforward input or relayed by first-order cells. This mechanism may reinforce a smoothing of iso-orientation domains except at points/frontiers of singularities in the orientation/direction preference maps, such as pinwheel centers or the borders of unidirectional domains. At such cortical locations, small changes in the relative contribution of some of the local sources may produce dramatic changes in the output preference of the cell. The non-homogeneities that we found in the relative orientation distribution of local and lateral inputs to a single cortical cell might indeed be the substrate of the anisotropy of adaptation-induced orientation plasticity observed in the orientation map using both optical imaging and electrophysiological techniques [21]. We conclude that examining the single cell spiking activity does not entirely reflect the properties of the cell's input receptive field, but that the combined knowledge of the contextual position in the global orientation network and of the spatial extent of the subthreshold receptive field is needed to identify the underlying integrative processes.

\section{Acknowledgements}

The cortical experiments described here were funded by grants from HFSP RG-10398, CNRS (CTI01-5), the Life-Like Perception program of the F.E.T initia- 
tive of the European Community (IST-2001-34712) to Y.F. We thank Dr. Andrew Davison for his helpful comments.

\section{References}

[1] P. Adojarn, J.B. Levitt, J.S. Lund, K. Obermayer, A model for the intracortical origin of orientation preference and tuning in macaque striate cortex, Visual Neurosci. 16 (2) (1999) 303-318.

[2] J.S. Anderson, M. Carandini, D. Ferster, Orientation tuning of input conductance, excitation, and inhibition in cat primary visual cortex, J. Neurophysiol. 84 (2) (2000) 909-926.

[3] J.S. Anderson, I. Lampl, D.C. Gillespie, D. Ferster, The contribution of noise to contrast invariance of orientation tuning in cat visual cortex, Science 290 (2000) 1968-1972.

[4] R. Ben-Yishai, R.L. Bar-Or, H. Sompolinsky, Theory of orientation tuning in visual cortex, Proc. Natl. Acad. Sci. USA 92 (9) (1995) 3844-3848.

[5] N.J. Berman, R.J. Douglas, K.A.C. Martin, D. Whitteridge, Mechanisms of inhibition in cat visual cortex, J. Physiol. 440 (1991) 697-722.

[6] L. Borg-Graham, C. Monier, Y. Frégnac, Voltage-clamp measurement of visually evoked conductances with whole-cell patch recordings in primary visual cortex, J. Physiol. (Paris) 90 (3-4) (1996) 185-188.

[7] L.J. Borg-Graham, C. Monier, Y. Frégnac, Visual input evokes transient and strong shunting inhibition in visual cortical neurons, Nature 393 (1998) 369-373.

[8] V. Bringuier, F. Chavane, L. Glaeser, Y. Frégnac, Horizontal propagation of visual activity in the synaptic integration field of area 17 neurons, Science 283 (1999) 695-699.

[9] M. Carandini, D.J. Heeger, Summation and division by neurons in primate visual cortex, Science 264 (1994) 1333-1336.

[10] M. Carandini, D.J. Heeger, J.A. Movshon, Linearity and gain control in V1 simple cells, in: P. Ulinsky, E.G. Heeger, A. Peters (Eds.), Cerebral Cortex, Vol. 13: Models of Cortical Circuits, Kluwer Academic/Plenum, New York, 1999, pp. 401-443.

[11] B. Chapman, K.R. Zahs, M.P. Stryker, Relation of cortical cell orientation selectivity to alignment of receptive fields of the geniculocortical afferents that arborize within a single orientation column in ferret visual cortex, J. Neurosci. 11 (1991) 1347-1358.

[12] F. Chavane, C. Monier, V. Bringuier, P. Baudot, L. BorgGraham, J. Lorenceau, Y. Frégnac, The visual cortical association field: a Gestalt concept or a physiological entity, J. Physiol. (Paris) 94 (5-6) (2000) 333-342.

[13] S. Chung, D. Ferster, Strength and orientation tuning of the thalamic input to simple cells revealed by electrically evoked cortical suppression, Neuron 20 (1998) 1177-1189.

[14] J.M. Crook, Z.F. Kisvarday, U.T. Eysel, Evidence for a contribution of lateral inhibition to orientation tuning and direction selectivity in cat visual cortex: reversible inactivation of functionally characterized sites combined with neuroanatomical tracing techniques, Eur. J. Neurosci. 10 (1998) 2056-2075.

[15] A. Das, C.D. Gilbert, Topography of contextual modulations mediated by short-range interactions in primary visual cortex, Nature 399 (6737) (1999) 655-661.

[16] G.C. DeAngelis, I. Ohzawa, R.D. Freeman, Receptive-field dynamics in the central visual pathways, Trends Neurosci. 18 (10) (1995) 451-458.

[17] R.J. Douglas, K.A.C. Martin, D. Whitteridge, Selective responses of visual cortical cells do not depend on shunting inhibition, Nature 332 (1988) 642-644.

[18] R.J. Douglas, K.A.C. Martin, A functional microcircuit for cat visual cortex, J. Physiol. 440 (1991) 735-769.
[19] R.J. Douglas, C. Koch, M.A. Mahowald, K.A.C. Martin, H.H. Suarex, Recurrent excitation in neocortical circuits, Science 269 (1995) 981-985.

[20] R.J. Douglas, K.A.C. Martin, Neuronal circuits of the neocortex, Annu. Rev. Neurosci., in press.

[21] V. Dragoi, C. Rivadulla, M. Sur, Foci of orientation plasticity in visual cortex, Nature 411 (2001) 80-86.

[22] D. Ferster, B. Jagadeesh, EPSP-IPSP interactions in cat visual cortex studied with in vivo whole-cell patch recording, J. Neurosci. 12 (4) (1992) 1262-1274.

[23] D. Ferster, S. Chung, H. Wheat, Orientation selectivity of thalamic input to simple cells of cat visual cortex, Nature 380 (1996) 249-252.

[24] D. Ferster, K.D. Miller, Neural mechanisms of orientation selectivity in the visual cortex, Annu. Rev. Neurosci. 23 (2000) $441-472$.

[25] J.A. Hirsch, J.M. Alonso, R. Clay Reid, L.M. Martinez, Synaptic integration in striate cortical simple cells, J. Neurosci. 18 (22) (1998) 9517-9528.

[26] J.A. Hirsch, L.M. Martinez, C. Pitlai, J.M. Alonso, Q. Wang, F.T. Sommer, Functionally distinct inhibitory neurons at the first stage of visual cortical processing, Nat. Neurosci. 6 (12) (2003) 1300 1308.

[27] D.H. Hubel, T.N. Wiesel, Receptive fields, binocular interaction and functional architecture in the cat's visual cortex, J. Physiol. 160 (1962) 106-154.

[28] C. Koch, T. Poggio, The synaptic veto mechanism: does it underlying direction and orientation selectivity in the visual cortex, in: D. Rose, V.G. Dobson (Eds.), Models of the Visual Cortex, J. Wiley and Sons, 1985, pp. 408-419.

[29] C. Koch, R. Douglas, U. Wehmeier, Visibility of synaptically induced conductance changes: theory and simulations of anatomically characterized cortical pyramidal cells, J. Neurosci. 10 (1990) $1728-1744$.

[30] T.Z. Lauritzen, K.D. Miller, Different roles for simple-cell and complex-cell inhibition, J. Neurosci. 23 (32) (2003) 10201-10213.

[31] L.M. Martinez, J.M. Alonso, R.C. Reid, J.A. Hirsch, Laminar processing of stimulus orientation in cat visual cortex, J. Physiol. 540 (2002) 321-333.

[32] D. McLaughlin, R. Shapley, M. Shelley, D.J. Wielaard, A neuronal network model of macaque primary visual cortex (V1): orientation selectivity and dynamics in the input layer 4Calpha, Proc. Natl. Acad. Sci. USA 97 (14) (2000) 8087-8092.

[33] C. Monier, F. Chavane, P. Baudot, L. Graham, Y. Frégnac, Orientation and direction selectivity of excitatory and inhibitory inputs in visual cortical neurons: a diversity of combinations produces spike tuning, Neuron 37 (4) (2003) 663-680.

[34] M.C. Morrone, D.C. Burr, L. Maffei, Functional implications of cross-orientation inhibition of cortical visual cells. I. Neurophysiological evidence, Proc. Roy. Soc. London 216 (1982) 335-354.

[35] S. Nelson, L. Toth, B. Sheth, M. Sur, Orientation selectivity of cortical neurons during intracellular blockade of inhibition, Science 265 (5173) (1994) 774-777.

[36] D. Pinto, J. Brumberg, D.J. Simons, Circuit dynamics and coding strategies in rodent somatosensory cortex, J. Neurophysiol. 83 (2000) 1158-1166.

[37] R.C. Reid, J.M. Alonso, Specificity of monosynaptic connections from thalamus to visual cortex, Nature 378 (1995) 281-284.

[38] J. Schummers, J. Marino, M. Sur, Synaptic integration by V1 neurons depends on location within the orientation map, Neuron 36 (2002) 969-978.

[39] G. Sclar, R.D. Freeman, Orientation selectivity in the cat's striate cortex is invariant with stimulus contrast, Exp. Brain Res. 46 (1982) $457-461$.

[40] R. Shapley, M. Hawken, D.L. Ringach, Dynamics of orientation selectivity in the primary visual cortex and the importance of cortical inhibition (Review), Neuron 38 (2003) 689-699. 
[41] A.M. Sillito, Inhibitory mechanisms influencing complex cell orientation selectivity and their modification at high resting discharge levels, J. Physiol. 289 (1979) 33-53.

[42] A.M. Sillito, J.A. Kemp, J.A. Milson, N. Berardi, A re-evaluation of the mechanism underlying simple cell orientation selectivity, Brain Res. 194 (1980) 517-520.

[43] B.C. Skottun, A. Bradley, G. Sclar, I. Ohzawa, R. Freeman, The effects of contrasts on visual orientation and spatial frequency discrimination: a comparison of single cells and behavior, J. Neurophysiol. 57 (1987) 773-786.

[44] D.C. Somers, E.V. Todorov, A.G. Siapas, L.J. Toth, D.S. Kim, M. Sur, A local circuit approach to understanding integration of long-range inputs in primary visual cortex, Cereb. Cortex 8 (3) (1998) 204-217.

[45] S. Thorpe, D. Fize, C. Marlot, Speed of processing in the human visual system, Nature 381 (1996) 520-522.

[46] T.W. Troyer, A.E. Krukowski, N.J. Priebe, K.D. Miller, Contrastinvariant orientation tuning in cat visual cortex: thalamocortical input tuning and correlation-based intracortical connectivity, J. Neurosci. 18 (1998) 5908-5927.

[47] F. Wörgötter, Comparing different modeling approaches of visual cortical cell characteristics, in: P.S. Ulinski, E.G. Jones, A. Peters (Eds.), Cerebral Cortex: Models of Cortical Circuits, Plenum Press, 1999, pp. 201-249. 